\title{
Mass Transfer Limitations of Porous Silicon-Based Biosensors for Protein Detection
}

\author{
Sofia Arshavsky Graham, Evgeniy Boyko, Rachel Salama, and Ester Segal* \\ Cite This: ACS Sens. 2020, 5, 3058-3069 \\ Read Online
}

ABSTRACT: Porous silicon (PSi) thin films have been widely studied for biosensing applications, enabling label-free optical detection of numerous targets. The large surface area of these biosensors has been commonly recognized as one of the main advantages of the PSi nanostructure. However, in practice, without application of signal amplification strategies, PSi-based biosensors suffer from limited sensitivity, compared to planar counterparts. Using a theoretical model, which describes the complex mass transport phenomena and reaction kinetics in these porous nanomaterials, we reveal that the interrelated effect of bulk and

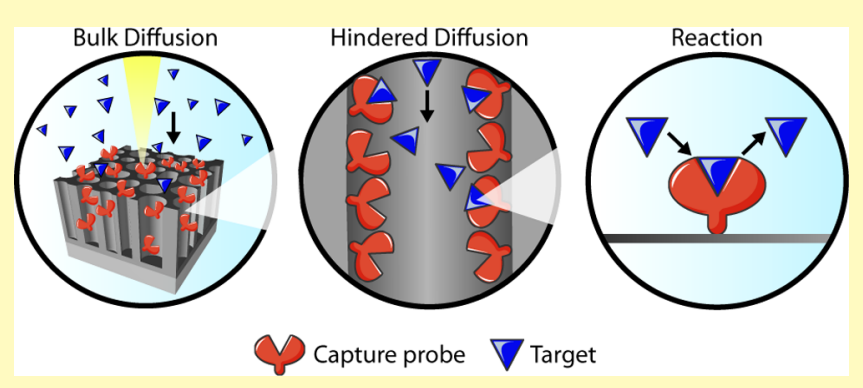
hindered diffusion is the main limiting factor of PSi-based biosensors. Thus, without significantly accelerating the mass transport to and within the nanostructure, the target capture performance of these biosensors would be comparable, regardless of the nature of the capture probe-target pair. We use our model to investigate the effect of various structural and biosensor characteristics on the capture performance of such biosensors and suggest rules of thumb for their optimization.

KEYWORDS: porous silicon, biosensors, aptasensor, mass transfer, hindered diffusion, aptamers, proteins

$\mathrm{B}$ iosensors that monitor the binding between a target molecule and a capture probe, by various transducing methods and surface-based detection, in which the capture probes are immobilized on the transducing surface, are among the most widespread bioanalytical tools. ${ }^{1-5}$ The performance of planar biosensors based on surface capture is governed by the complex interplay between transport phenomena and reaction kinetics, as modeled by Squires et al. ${ }^{6}$ As such, numerous studies have been directed to optimize these systems and elucidate their limiting factors. ${ }^{7-14}$

Porous silicon (PSi) has been widely studied as an optical transducing surface in various biosensing platforms, presenting low cost fabrication, chemically active surface, and unique optical properties. ${ }^{15,16}$ Specifically, employing interferometric Fourier transform spectroscopy (RIFTS) as the transduction mechanism enables real-time and label-free target detection. ${ }^{17-20}$ In this method, a series of Fabry-Pérot interference fringes are produced from incident white light reflections from the top and bottom interfaces of a porous thin film, and the fringe pattern depends on the thickness and averaged refractive index of the porous layer.

The porous nanostructure of PSi increases the surface area dramatically, which allows the immobilization of a greater amount of capture probe molecules compared to planar devices and potentially increases the detection sensitivity by orders of magnitude. ${ }^{16,21-25}$ Nevertheless, common detection thresholds in such systems revealed an inferior performance, with micromolar detection limits for protein and DNA targets in direct and label-free optical detection. ${ }^{15,22,25-29}$ Therefore, many have focused on developing assays for improving the sensitivity and performance of such systems, ${ }^{15,26,27,29-31}$ while others investigated the limiting characteristics of the platform and suggested solutions for overcoming these issues. ${ }^{28,32-34}$ The latter includes mass transfer limitations, which are affected by the nanostructure characteristics such as pore size, height, porosity, surface area, and roughness. ${ }^{32-40}$ For example, studies on the impact of the pore size on the binding efficiency have been conducted, ${ }^{39,41}$ and a critical correlation between the molecule size and the pore diameter has been suggested to allow effective infiltration into the porous layer, in which the molecule must be at least five times smaller than the pore opening. ${ }^{33}$ To overcome mass transport limitations, flowthrough platforms have been developed. ${ }^{28,42}$ Moreover, accurate quantitative determination of molecular binding kinetics was performed by analyzing dilute analyte solution at short binding times, avoiding capture probe saturation with the analyte. ${ }^{43}$ The many parameters affecting the performance of PSi biosensors challenge the experimental characterization of each factor. Thus, deriving theoretical models to describe

Received: April 2, 2020

Accepted: September 8, 2020

Published: September 8, 2020 
Table 1. Properties of the Studied Capture Probe and Target Protein Pairs and Comparison of the Theoretical and Fitted $K_{\mathrm{D}}$ Values

\begin{tabular}{|llc}
\hline aptamer/antibody & \multicolumn{1}{c}{ target } & molecular weight $(\mathrm{kD})$ \\
\hline anti-his tag aptamer & $\mathrm{D} 2$ & 60 \\
anti-his tag aptamer & tyrosinase & 35 \\
anti-his tag antibody & tyrosinase & 35 \\
anti-AGR2 aptamer & AGR2 & 22 \\
anti-protein A aptamer & protein A & 45 \\
\hline
\end{tabular}

the effect of each of the parameters is a facile way to study these complex systems.

In mesoporous systems, as the size of the pore approaches that of the solute, a deviation from the diffusion kinetics predicted by Fick's law is observed, leading to an overestimation of the solute flux. ${ }^{44-46}$ The diffusion within the porous nanostructure is hindered by steric and hydrodynamic interactions between the diffusing solute, the pore wall, and the immobilized molecules and receptors on the pore wall. ${ }^{44,47-50}$ The structural properties of the porous nanostructure, such as the pore diameter and porous layer thickness, have also a tremendous effect, as the deviation from the bulk diffusion coefficient is more pronounced for smaller pores and a thicker layer. ${ }^{51}$ The motion of the diffusing molecule is also highly dependent on structural defects, as revealed by single-molecule diffusion analysis in mesoporous silica. ${ }^{52,53}$ Molecule transport and adsorption in porous materials have been investigated, and models describing the hindered diffusion effect have been empirically derived. ${ }^{46,54}$ Nevertheless, the spatial and timeresolved change in the diffusivity coefficient upon the filling of the pore has been often neglected to simplify the solution of the mass balances. ${ }^{55-57}$

Further simplification of the mass transfer studies in porous biosensors has approximated the porous layer as a perfect collector, given the large capacity of binding sites within the porous layer. Thus, the entry into the pores has been concluded as the rate-limiting step, while the hindered diffusion effect has been neglected. ${ }^{28,58,59}$ Such modeling and analysis were derived for protein adsorption on a porous anodic aluminum oxide nanostructure ${ }^{58}$ and for PSi-based optical biosensors. $^{28,59}$ Nevertheless, for evaluation of the effect of mass transfer or reaction kinetics in PSi-based biosensors, the system cannot be assumed as a planar surface, and all transport phenomena and reaction between the target and the immobilized probes should be considered.

In this work, we aim to determine the effect of each of the mass transport phenomena and reaction kinetics in PSi-based optical biosensors. In contrast to previous studies, we develop a model which captures the complex mass transfer processes in porous materials, including bulk diffusion, hindered diffusion, and capture probe-target binding kinetics. The model is solved numerically using parameters which are characteristic of typical PSi biosensors. Specifically, we compare our model results to biosensing experiments of several PSi-based aptasensors for proteins targets, as well as to the common simplified "planar" model, which neglects the hindered diffusion within the porous layer. Importantly, we determine the limiting transport phenomena of PSi-based biosensors and the dependency of the target binding rate on various biosensor characteristics and conclude with directions for proper optimization of such biosensors.

$\begin{array}{lc}\text { literature } K_{\mathrm{D}} & \text { fitted } K_{\mathrm{D}}(\mu \mathrm{M}) \\ \sim 4.6 \mu \mathrm{M}^{63} & 29 \pm 8\left(R^{2}=0.9551\right) \\ \sim 4.6 \mu \mathrm{M}^{63} & 31 \pm 7\left(R^{2}=0.9731\right) \\ \sim 10 \mathrm{nM}^{64} & 24 \pm 5\left(R^{2}=0.9420\right) \\ \sim 13 \mathrm{nM}^{60} & 21 \pm 1\left(R^{2}=0.9951\right) \\ \sim 0.522 \mu \mathrm{M}^{62} & 14 \pm 1\left(R^{2}=0.9177\right)\end{array}$

\section{MATERIALS AND METHODS}

Materials. Heavily doped p-type Si wafers $(\langle 100\rangle$-oriented, 0.90$1.00 \mathrm{~m} \Omega \cdot \mathrm{cm}$ resistivity) were purchased from Sil'tronix Silicon Technologies (France). Aqueous HF (48\%), (3-aminopropyl)triethoxysilane (APTES), ethyldiisopropylamine (DIEA), succinic acid, succinic anhydride, $\mathrm{N}$-(3-dimethylaminopropyl)- $\mathrm{N}^{\prime}$-ethylcarbodiimide hydrochloride (EDC), N-hydroxysuccinimide (NHS), glutaraldehyde $25 \%$ solution (GA), ethanolamine, acetonitrile (ACN), dimethyl sulfoxide (DMSO), sodium cyanoborohydride, sodium dodecyl sulfate (SDS), $\mathrm{N}$-succinimidyl 3-(2-pyridyldithio)propionate (SPDP), DL-dithiothreitol (DTT), isopropyl alcohol (IPA), morpholinoethanesulfonic acid (MES), MES sodium salt, Tris base, and all buffer salts were purchased from Merck (Israel). Ethanol absolute was supplied by Bio-Lab ltd (Israel). All solutions were prepared with Milli-Q water $\left(\mathrm{ddH}_{2} \mathrm{O}, 18.2 \mathrm{M} \Omega \cdot \mathrm{cm}\right)$. The antiAGR2 aptamer sequence ( 5 '-TCT-CGG-ACG-CGT-GTG-GTCGGG-TGG-GAG-TTG-TGG-GGG-GGG-GTG-GGA-GGG-TT-3' ) was obtained from $\mathrm{Wu}$ et al. ${ }^{60}$ The anti-his tag aptamer $6 \mathrm{H} 7$ sequence (5'-GCT ATG GGT GGT CTG GTT GGG ATT GGC CCC GGG AGC TGG C-3') was taken from U.S. patent $7329742 .{ }^{61}$ These aptamers were purchased with a $5^{\prime}$-amino modification from Integrated DNA Technologies (USA). The anti-protein A aptamer, selected by Stoltenburg et al., ${ }^{62}$ was used in the truncated form PA\#2/ 8[S1-58]: 5'-ATA CCA GCT TAT TCA ATT AGC AAC ATG AGG GGG ATA GAG GGG GTG GGT TCT CTC GGC T-3' and purchased with $3^{\prime}$-amino-C6 modification. AGR2 protein was purchased from MyBioSource Inc (USA). The mouse anti-his monoclonal antibody was obtained from Enco (Israel). Streptavidin, biotinylated protein $\mathrm{A}$, and recombinant protein $\mathrm{A}$ from human serum were purchased from Merck (Israel). 6xhis tyrosinase from Bacillus megatherium (recombinant, expressed in Escherichia coli) was generously supplied by Prof. Ayelet Fishman, Technion. His-tagged AbnA-D2 (from Geobacillus stearothermophilus T-6, expressed in E. coli) (D2) was generously supplied by Prof. Yuval Shoham, Technion. Phosphate-buffered saline (PBS, $10 \mathrm{mM}$ ) was composed of $137 \mathrm{mM}$ $\mathrm{NaCl}, 2.7 \mathrm{mM} \mathrm{KCl}, 10 \mathrm{mM} \mathrm{Na}_{2} \mathrm{HPO}_{4}$, and $2 \mathrm{mM} \mathrm{KH}_{2} \mathrm{PO}_{4}(\mathrm{pH} 7.5$ ). AGR2 selection buffer was composed of $137 \mathrm{mM} \mathrm{NaCl}, 20 \mathrm{mM} \mathrm{KCl}$, $10 \mathrm{mM} \mathrm{Na}_{2} \mathrm{HPO}_{4}$, and $2 \mathrm{mM} \mathrm{KH}_{2} \mathrm{PO}_{4}$ (pH 7.4). $6 \mathrm{H} 7$ selection buffer was composed of $50 \mathrm{mM} \mathrm{K}_{2} \mathrm{HPO}_{4}$ and $150 \mathrm{mM} \mathrm{NaCl}$, pH 7.4, and elution buffer was composed of $50 \mathrm{mM} \mathrm{K}_{2} \mathrm{HPO}_{4}, 150 \mathrm{mM} \mathrm{NaCl}$, and $1 \mathrm{M}$ Imidazole ( $\mathrm{pH}$ 7.4). Protein A aptamer selection buffer was composed of $20 \mathrm{mM}$ Tris base, $100 \mathrm{mM} \mathrm{NaCl}, 5 \mathrm{mM} \mathrm{KCl}, 10 \mathrm{mM}$ $\mathrm{MgCl}_{2}$, and $1 \mathrm{mM} \mathrm{CaCl} 2$. MES buffer $(0.5 \mathrm{M})$ was prepared from $0.27 \mathrm{M}$ MES and 0.23 M MES sodium salt (pH 6.1), and Tris buffer was composed of $50 \mathrm{mM}$ Tris base ( $\mathrm{pH} 7.4$ ).

Construction of PSi-Based Biosensors. The studied biosensors include several PSi-based aptasensors and a representative immunosensor, as detailed in Table 1. All biosensors employed a similar PSi nanostructure as the optical transducer, and capture probe molecules (aptamer or antibody) were immobilized onto the surface.

PSi Fabrication. PSi Fabry-Pérot thin films are fabricated from highly doped p-type crystalline $S i$ wafers, with a typical resistivity of 0.90-1.00 $\mathrm{m} \Omega \cdot \mathrm{cm}$, using a two-step anodic electrochemical etching process. A detailed description of the etching setup can be found elsewhere. ${ }^{18}$ First, a sacrificial layer is etched at a constant current density of $300 \mathrm{~mA} \mathrm{~cm}^{-2}$ for $30 \mathrm{~s}$ for the anti-AGR2 system or $375 \mathrm{~mA}$ $\mathrm{cm}^{-2}$ for $30 \mathrm{~s}$ for the other systems, in a 3:1 (v/v) solution of aqueous HF $(48 \%)$ and ethanol, respectively. The obtained porous layer is removed by introduction of $0.1 \mathrm{M} \mathrm{NaOH}$, followed by exposure to a 
solution of 1:3:1 (v/v) HF, ethanol, and $\mathrm{ddH}_{2} \mathrm{O}$, respectively. Next, a second etching is performed, under the same etching conditions as mentioned above. After each step, the silicon surface is thoroughly rinsed with ethanol and dried under a nitrogen stream. Subsequently, the freshly etched PSi is thermally oxidized in a tube furnace (Thermo Scientific, Lindberg/Blue M $1200{ }^{\circ} \mathrm{C}$ Split-Hinge, USA) at $800{ }^{\circ} \mathrm{C}$ for $1 \mathrm{~h}$ in ambient air to create a chemically stable and hydrophilic oxidized PSi scaffold. ${ }^{65}$

Aptamer Immobilization. Amino-terminated aptamers are conjugated to the oxidized PSi films by carbodiimide coupling chemistry. The first two steps of the chemistry, amino-silanization and carboxylation, slightly differ in solvents and materials for each sensing system. For anti-AGR2 aptamer immobilization, the oxidized PSi film is amino-silanized by incubation in $1 \% \mathrm{v} / \mathrm{v}$ APTES and $1 \% \mathrm{v} / \mathrm{v}$ DIEA in $\mathrm{ddH}_{2} \mathrm{O}$ solution for $1 \mathrm{~h}$, followed by washing with $\mathrm{ddH}_{2} \mathrm{O}$ and ethanol and drying under a nitrogen stream. Subsequently, the aminoactivated PSi samples are annealed at $100{ }^{\circ} \mathrm{C}$ for $15 \mathrm{~min}$. Next, carboxylation is achieved by incubation in a solution of succinic anhydride $\left(10 \mathrm{mg} \mathrm{mL}^{-1}\right)$ and $2 \% \mathrm{v} / \mathrm{v}$ DIEA in ACN for $3 \mathrm{~h}$, followed by extensive rinsing with $\mathrm{ACN}$ and $\mathrm{ddH}_{2} \mathrm{O}$ and drying under a nitrogen stream.

The anti-his tag aptamer, $6 \mathrm{H} 7$, and the anti-protein A aptamer are immobilized by the method described by Urmann et al. ${ }^{66,67}$ Briefly, the oxidized PSi films are reacted with a solution of $42 \mathrm{mM}$ APTES in toluene for $1 \mathrm{~h}$, followed by a thorough rinsing with toluene, ethanol, and acetone and drying under a nitrogen stream. A similar annealing step is then performed, as described above. The APTES-modified surface is then incubated in a solution of $100 \mathrm{mg}$ of succinic acid in $4.7 \mathrm{~mL}$ of DMSO and $300 \mu \mathrm{L}$ of $0.1 \mathrm{M} \mathrm{NaHCO}_{3}$ (pH 9.4) for 30 min, followed by washing with DMSO and $\mathrm{ddH}_{2} \mathrm{O}$ and drying under a stream of nitrogen.

Subsequently, for both systems, the carboxylated samples are reacted with EDC $\left(10 \mathrm{mg} \mathrm{mL} \mathrm{m}^{-1}\right)$ in the corresponding selection buffer for $1 \mathrm{~h}$, followed by introduction of $50 \mu \mathrm{M}$ anti-AGR2 aptamer or $75 \mu \mathrm{M}$ anti-his tag or anti-protein $\mathrm{A}$ aptamers in selection buffer and incubation for $1 \mathrm{~h}$. The samples are then washed with Tris buffer to deactivate the remaining reactive EDC groups on the surface. Finally, the aptamer-functionalized PSi is exposed to boiling $\mathrm{ddH}_{2} \mathrm{O}$ for 2 min and gently dried under a nitrogen stream to unfold any secondary structures of the aptamer prior to further use.

Antibody Immobilization. The oxidized PSi surface is first aminosilanized in $1 \% \mathrm{v} / \mathrm{v}$ APTES and $1 \% \mathrm{v} / \mathrm{v}$ DIEA in $\mathrm{ddH}_{2} \mathrm{O}$ solution for 1 $\mathrm{h}$, followed by washing with $\mathrm{ddH}_{2} \mathrm{O}$ and ethanol and drying under a nitrogen stream. Subsequently, the surface is exposed to $2 \%$ aqueous GA solution for $30 \mathrm{~min}$, washed with $\mathrm{ddH}_{2} \mathrm{O}$, and dried under a nitrogen stream, followed by incubation with $50 \mathrm{mM}$ sodium cyanoborohydride in HEPES for $30 \mathrm{~min}$, in order to stabilize the Schiff base formed during reaction of the aldehyde groups with the amine groups. ${ }^{68}$ Next, the surface is washed with HEPES, and streptavidin $\left(100 \mu \mathrm{L} ; 100 \mu \mathrm{g} \mathrm{mL}^{-1}\right.$ in PBS) is applied and incubated for $1 \mathrm{~h}$. The surface is washed with PBS and stabilized again with sodium cyanoborohydride. Next, a blocking step with $0.3 \mathrm{M}$ ethanolamine in BBS buffer $(0.15 \mathrm{M}$ borate buffered saline, $\mathrm{pH}$ 9.0) is carried out for $30 \mathrm{~min}$, followed by washing with BBS and PBS buffers. Finally, the surface is reacted with biotinylated protein A (100 $\mu \mathrm{L} ; 100 \mu \mathrm{g} \mathrm{mL}^{-1}$ in PBS) for $1 \mathrm{~h}$, rinsed with PBS, and incubated with the antibody $\left(50 \mu \mathrm{L} ; 100 \mu \mathrm{g} \mathrm{mL} \mathrm{m}^{-1}\right.$ in PBS; in humidity chamber) for $1 \mathrm{~h}$ at room temperature and then overnight at $8{ }^{\circ} \mathrm{C}$. On the next day, the film is rinsed with PBS buffer prior to biosensing experiments.

Determination of Aptamer Concentration within PSi. Quantification of the immobilized aptamer concentration is carried out by the method described by $\mathrm{Hu}$ et al. ${ }^{23}$ for the anti-AGR2 and anti-his tag aptasensors. We used aptamers with a thiol and FAM6 modification which are diluted in TE buffer $(10 \mathrm{mM}$ Tris and $1 \mathrm{mM}$ EDTA, pH 8.0), supplemented with $30 \mathrm{mM}$ DTT. Prior to use, the aptamer is cleaned in a NAP-5 column (GE healthcare), in HEPES buffer (0.05 M HEPES, pH 7.5) to remove the DTT reducing agent. After amino-silanization of the oxidized PSi films, the samples are reacted with SPDP (6.5 $\mathrm{mM}$ in ethanol) for $30 \mathrm{~min}$, followed by washing with IPA and $\mathrm{ddH}_{2} \mathrm{O}$ three times. Anti-AGR2 $(50 \mu \mathrm{M})$ or 75 $\mu \mathrm{M} 6 \mathrm{H} 7$ aptamers in HEPES buffer are then introduced to the samples and incubated for $1 \mathrm{~h}$, followed by extensive washing with HEPES, to remove physisorbed aptamer molecules. As a control, oxidized PSi is similarly functionalized with SPDP, but without the aptamer. The surface is washed until no fluorescence signal is detected in the collected washing solution, compared to the control.

For the anti-AGR2 aptamer, the aptamer-functionalized PSi is incubated with DTT solution $(250 \mu \mathrm{L} ; 25 \mathrm{mM}$ in HEPES, pH 7.5) for $30 \mathrm{~min}$, resulting in immediate aptamer cleavage from the surface by disulfide bond reduction. The cleaved aptamer solution is collected, and the absorbance is measured at $495 \mathrm{~nm}$ using a plate reader (Thermo Scientific Varioskan), as described by $\mathrm{Hu}$ et al. ${ }^{23}$ For the anti-his tag aptamer, the aptamer cleavage from the surface is slower, attributed to the different amino silanization procedures. Thus, the aptamer-functionalized surface is incubated with reducing solution for $24 \mathrm{~h}$, followed by solution collection and replacement with a fresh reducing solution. This process is repeated until no fluorescence signal is observed in the collected solution (compared to the control). The fluorescence of the collected solutions is analyzed using a plate reader at excitation and emission wavelength values of 490 and $525 \mathrm{~nm}$, respectively, enabling more sensitive determination of the slower cleavage process. The measured absorbance or fluorescence values are correlated to the respective aptamer concentrations using a calibration curve, which is constructed using known concentrations of the FAM6-labeled aptamer (in $25 \mathrm{mM}$ DTT in HEPES buffer).

Biosensing Experiments. Protein Targets. A $60 \mathrm{kDa}$ his-tagged protein from the Arabinase family, named D2, and a $35 \mathrm{kDa}$ histagged tyrosinase from $B$. megatherium are used as targets for the antihis tag $6 \mathrm{H} 7$ aptamer-functionalized $\mathrm{PSi}^{66}{ }^{66}$ The his-tagged tyrosinase is also detected by an anti-his tag antibody-functionalized PSi. The 45 $\mathrm{kDa}$ recombinant protein $\mathrm{A}$ from $S$. aureus is used as a target for the anti-protein A aptamer, ${ }^{67}$ and the $22 \mathrm{kDa}$ AGR2 protein is detected by the anti-AGR2 aptamer-functionalized PSi.

Optical Setup. The RIFTS method is utilized for real-time monitoring of changes occurring within the porous nanostructure by detection of variations in the average refractive index of the porous layer. ${ }^{19,20,66}$ The aptamer- or antibody-functionalized PSi sample is mounted in a custom-made Plexiglas cell, which is fixed during the experiments to ensure that the reflectivity is measured at the same spot throughout the experiment. Interferometric reflectance spectra are collected with a charge-coupled device (CCD) spectrometer (Ocean Optics, USB 4000) fitted with an objective lens coupled to a bifurcated fiber-optic cable. A tungsten light source is focused onto the center of the sample with a spot size of approximately $1 \mathrm{~mm}^{2}$. Illumination and reflectivity detection are performed perpendicular to the surface. Reflectivity spectra are collected in real time in a wavelength range of $450-900 \mathrm{~nm}$ and analyzed by applying fast Fourier transformation (FFT), as previously described by MassadIvanir et al. ${ }^{69}$ The latter results in a single peak, which positions along the $x$-axis, equals the effective optical thickness (EOT) of the porous layer and is the product of the average refractive index and the thickness of the porous layer.

Experimental Procedure. For the anti-his tag biosensor, the surface is first washed with elution buffer for $30 \mathrm{~min}$ to unfold the aptamer. Then and for all biosensing systems, the PSi biosensor is incubated with the baseline buffer, the corresponding selection buffer for the aptasensors or PBS for the immunosensor, for at least 30 min until a stable baseline is acquired. Next, the target protein, diluted in the baseline buffer, is introduced and allowed to incubate for $1 \mathrm{~h}$ or until a steady-state signal is obtained. Subsequently, the biosensor is extensively washed with the baseline buffer. Throughout the experiment, the reflectivity spectra are recorded every $15 \mathrm{~s}$, while during buffer exchange and rinsing steps, reflectivity measurements are shortly paused.

Data Analysis. Reflectivity data are presented as relative $\Delta \mathrm{EOT}$, defined as 

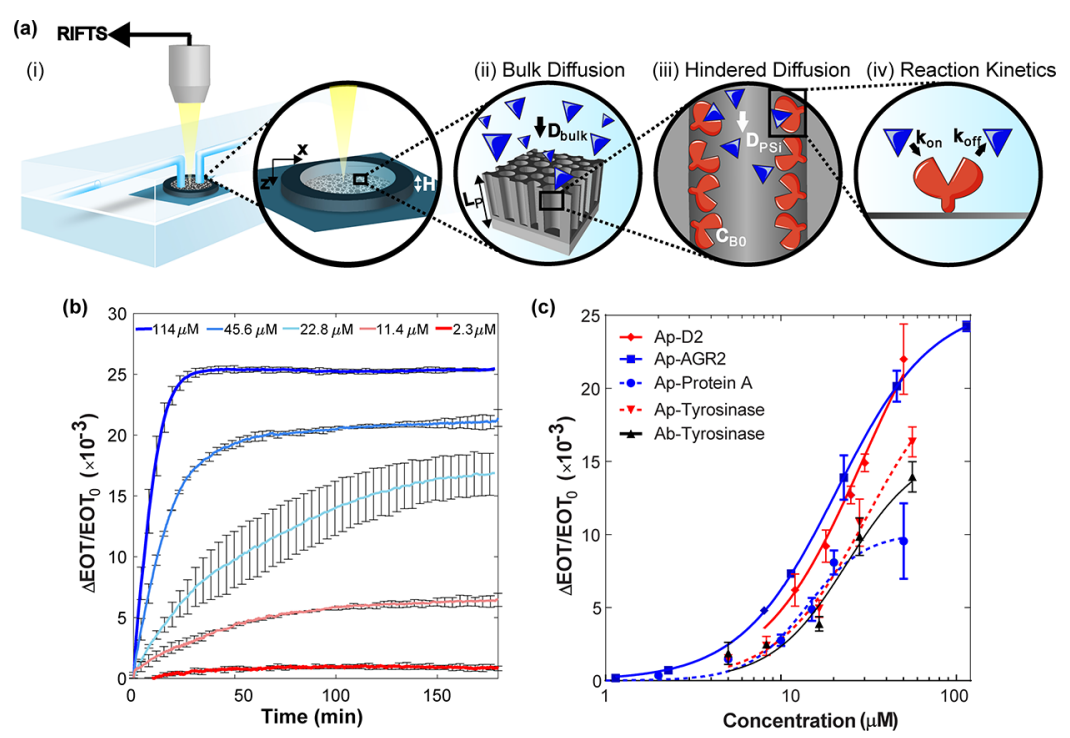

Figure 1. PSi biosensor setup and characteristic biosensing results for different capture probe-target pairs. (a) Schematic illustration of the PSi biosensing system: (i) Traditional cell setup used for the RIFTS biosensing experiments. The PSi-based biosensor is fixed in the cell with an O-ring, confining the introduced solution to a height of $H$. (ii) Target solution is introduced to the cell, and target proteins diffuse to the PSi biosensor with diffusivity $D_{\text {bulk }}$. (iii) As arriving to the pore entry, the proteins diffuse inside the porous nanostructure with diffusivity $D_{\mathrm{PSi}}$. The PSi is functionalized with capture probe molecules at a concentration of $c_{\mathrm{B} 0}$, and the porous layer thickness is $L_{\mathrm{p}}$. (iv) While diffusing, the target binds to the immobilized capture probe with kinetic parameters of $k_{\text {on }}$ and $k_{\text {off }}$ (b) Characteristic biosensing results presenting the relative EOT changes with time for anti-AGR2 aptasensors upon incubation with different concentrations of AGR2 protein solutions $(n \geq 3)$. The relative EOT increases with the infiltration and diffusion of the target protein into the porous layer, followed by binding to the immobilized aptamer probes. (c) Binding curves of different protein targets on aptamer (Ap)- or antibody (Ab)-functionalized PSi-based biosensors, fitted with a specific binding model with a Hill slope. The curves present a similar behavior, independent of the target protein, capture probe, and their corresponding theoretical binding affinity.

$$
\frac{\Delta \mathrm{EOT}_{t}}{\mathrm{EOT}_{0}}=\frac{\mathrm{EOT}_{t}-\mathrm{EOT}_{0}}{\mathrm{EOT}_{0}}
$$

where $\mathrm{EOT}_{0}$ is the averaged EOT signal obtained during baseline establishment. For the binding curve, the $\mathrm{EOT}_{t}$ used is the averaged EOT signal at equilibration, following the wash of unbound proteins.

LOD is calculated as the protein concentration for which the optical signal equals $3 \cdot \sigma$, where $\sigma$ is the standard deviation between relative EOT values, measured during baseline establishment. Nonlinear regression of obtained data was performed with GraphPad Prism software utilizing the model for specific binding with a hill slope, according to

$$
Y=\frac{B_{\max } \cdot X^{\mathrm{h}}}{\left(K_{\mathrm{D}}^{\mathrm{h}} \cdot X^{\mathrm{h}}\right)}
$$

$B_{\max }$ is the interpolated concentration at which the maximum biosensor response is reached, and $K_{D}$ is the apparent dissociation constant, which is the target concentration needed to reach the halfmaximum biosensing signal. $h$ is the Hill coefficient, which gives information about the stoichiometry of the binding interaction. ${ }^{70,71}$ Table 1 summarizes the fitted $K_{\mathrm{D}}$ values.

Numerical Simulations. We performed numerical simulations of the governing equations using finite differences. We first discretized the spatial derivatives using a second-order central difference approximation with uniform grid spacing, leading to a series of coupled ordinary differential equations. We then integrated the resulting set of ordinary differential equations forward in time using Matlab's routine ode15s (Matlab R2018sb, MathWorks, Inc.). For the simulation, we used the parameters of the aptasensors, as summarized in Table S1 (Supporting Information): a height of the solution above the PSi of $0.001 \mathrm{~m}$, a porous layer thickness of $5.5 \times 10^{-6} \mathrm{~m}$, an average pore diameter of $50 \times 10^{-9} \mathrm{~m}$, a hydrodynamic radius of the analyte of $5.3 \times 10^{-9} \mathrm{~m}$, a hydrodynamic radius of the capture probe of $3.0 \times 10^{-9} \mathrm{~m}$, a protein bulk diffusivity of $7 \times 10^{-11} \mathrm{~m}^{2} \mathrm{~s}^{-1}$, a capture probe concentration within the PSi layer of $3.6 \times 10^{-3} \mathrm{M}$, a capture probe surface density of $1.2 \times 10^{-8} \mathrm{~mol} \mathrm{~m}^{-2}$, a reaction association rate of $1.21 \times 10^{3} \mathrm{M}^{-1} \mathrm{~s}^{-1}$, and a reaction dissociation rate of $6.32 \times 10^{-4} \mathrm{~s}^{-1}$. For higher affinity interaction simulation, a reaction association rate of $1 \times 10^{5} \mathrm{M}^{-1} \mathrm{~s}^{-1}$ and a reaction dissociation rate of $1 \cdot 10^{-4} \mathrm{~s}^{-1}$ are used.

\section{RESULTS AND DISCUSSION}

PSi-Based Biosensors for Protein Targets. PSi FabryPérot thin film-based biosensors are widely studied for detection of various target molecules. ${ }^{15,16}$ Over the past few years, we have established several such biosensors for detection of different protein targets ${ }^{66,67}$ (see Table 1), using both antibodies and aptamers as capture probes. All these biosensors are based on a similar oxidized PSi nanostructure ( $>70 \%$ porosity), which is $\sim 5 \mu \mathrm{m}$ thick and is characterized by interconnected cylindrical pores with an average diameter of $50 \mathrm{~nm},{ }^{66,67}$ where capture probe molecules (amino-terminated DNA aptamers or antibodies) are immobilized via different techniques. $^{66,67,72,73}$

Biosensing experiments are performed in a conventional cell setup, illustrated in Figure $1 \mathrm{a}(\mathrm{i})$, where the target protein solutions are introduced on the top of the biosensor and incubated (without convection). Figure $1 \mathrm{~b}$ presents characteristic biosensing results for an aptasensor upon incubation with different concentrations of the target protein, where the EOT changes are plotted as a function of time. As the target protein diffuses in the bulk solution toward the pore entry [Figure $1 \mathrm{a}(\mathrm{ii})]$, it infiltrates into the nanostructure, diffuses, and simultaneously interacts with the immobilized aptamer molecules [Figure 1a(iii,iv), respectively], resulting in an increase in the EOT signal with time. After the EOT signal reaches equilibrium, the biosensor is washed with buffer solution to remove nonbound target molecules, and the 


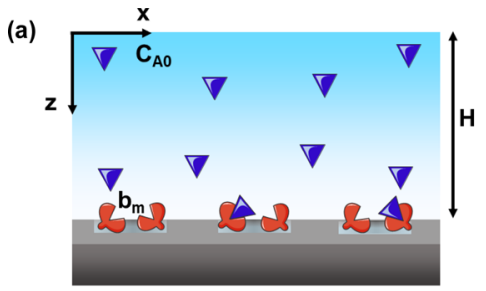

(b)

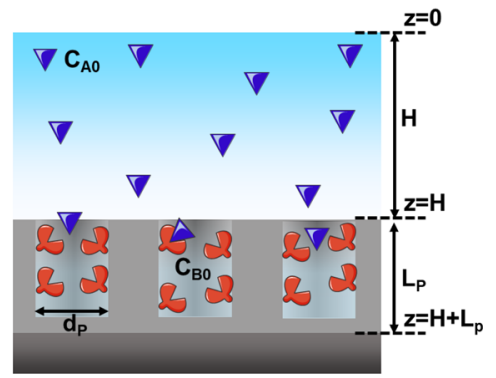

Figure 2. Schematic illustration of the (a) planar and (b) porous models, describing the mass transfer and reaction kinetic phenomena in the PSibased biosensors. The PSi has a thickness of $L_{\mathrm{p}}$ and an average pore diameter of $d_{\mathrm{p}}$. The solution height above the porous layer is $H$. Capture probes are immobilized at a concentration of $c_{\mathrm{B} 0}$ and a density of $b_{\mathrm{m}}$. The target analyte is introduced to the biosensor at a concentration of $c_{\mathrm{A} 0}$.

attained signal is used for constructing a binding curve. Figure 1c presents characteristic binding curves for several studied capture probe-target pairs and their corresponding curve fit, utilizing a model for specific binding with a Hill slope. ${ }^{70,71}$ Surprisingly, all the investigated biosensors present a similar performance, with a dynamic range in the lower micromolars and a measured limit of detection (LOD) of $\sim 1 \mu \mathrm{M}$, regardless of the nature of the capture probe, the target protein, and their binding affinity. Moreover, the apparent dissociation constant $\left(K_{\mathrm{D}}\right)$ values, as calculated from the binding curves, are in the range of $14-31 \mu \mathrm{M}$, where these values are significantly higher by few orders of magnitude from those reported in the literature, see Table 1 . These results may suggest that the major limiting factor of these biosensors is the porous platform and, specifically, the involved complex mass-transfer phenomena. As the binding behavior is similar, regardless of the theoretical affinity between the capture probe and the target, we hypothesize that the effect of reaction kinetics is less pronounced. However, as any measurement is limited by the experimental setup, the signal processing method, and, consequently, the noise of the system, these also play a role, as has been recently suggested by Barillaro and co-workers. They applied a different signal processing technique (named interferogram average over wavelength, IAW) instead of the common EOT calculation, which resulted in a significant improvement in the LOD of the PSi biosensors. ${ }^{26,27}$ However, in the present work, we focus on the fundamental mass transfer phenomena in PSi biosensors and study their effect on biosensing performance.

Mass Transfer and Reaction Kinetics Model. The theoretical models, which describe the mass transfer in porous biosensors, and specifically PSi-based biosensors, commonly apply a perfect collector assumption to the porous layer. ${ }^{28,58,59}$ As such, the rate-limiting step is assumed as the entry into the pores, while the diffusion within the pores is neglected and the porous surface is modeled as a flat capturing surface, with a capture probe surface density of $b_{\mathrm{m}}$, as schematically illustrated in Figure $2 \mathrm{a}^{28,58,74}$ The derivation of such a model, named in this work as the "planar model", is detailed in the Supporting Information. Our aim is to investigate a complete model, which includes both transport phenomena (to and within the pores) and reaction, as illustrated in Figure $2 \mathrm{~b}$. To this end, we formulate a one-dimensional model, termed in this work as the "porous model", describing the concentration of the target analyte as a function of time. We refer to a conventional cell setup with solution height $H$ above the PSi biosensor, a porous layer of thickness $L_{\mathrm{p}}$, and an average pore diameter of $d_{\mathrm{p}}$. For simplification, we assume that the pores are stacked to each other, and we neglect the interpore distance (consistent with the high porosity of the PSi). The concentration of the immobilized capture probe molecules and the introduced analyte are $c_{\mathrm{B} 0}$ and $c_{\mathrm{A} 0}$, respectively. We assume no convection and one-dimensional diffusion, directed in the $z$ axis only.

In the bulk solution, the time evolution of the analyte concentration, $c_{\mathrm{A}, \text { bulk }}(z, t)$, is governed by Fick's second law

$$
\frac{\partial c_{\mathrm{A}, \text { bulk }}}{\partial t}=D_{\text {bulk }} \frac{\partial^{2} c_{\mathrm{A}, \text { bulk }}}{\partial z^{2}} \quad 0<z<H
$$

Subscripts of the bulk refer to the bulk solution, where $D_{\text {bulk }}$ is the analyte diffusivity coefficient in the bulk solution.

Within the porous layer, we describe the time evolution of analyte concentration, $c_{\mathrm{A}, \mathrm{PSi}}(z, t)$, by the diffusion-reaction equation

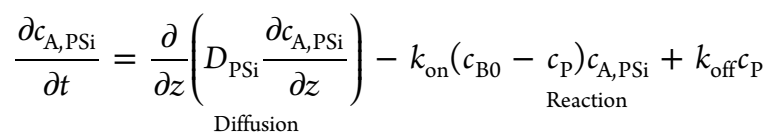

$$
\begin{aligned}
& H<z<H+L_{\mathrm{p}}
\end{aligned}
$$

Note that subscripts of PSi refer to the porous layer, where the analyte diffusivity coefficient is $D_{\mathrm{PSi}}$. This is the main difference with respect to the planar model, in which the diffusion within the porous layer is neglected and the reaction is only considered on the PSi surface as a boundary condition (see the Supporting Information).

We use the standard ligand-receptor model to describe the simultaneous reaction of the analyte with the immobilized capture probes, and accordingly, the concentration of the bound analyte-probe complexes, $c_{P}(z, t)$, evolves as

$$
\frac{\partial c_{\mathrm{P}}}{\partial t}=k_{\mathrm{on}}\left(c_{\mathrm{B} 0}-c_{\mathrm{P}}\right) c_{\mathrm{A}, \mathrm{PSi}}-k_{\mathrm{off}} \mathcal{c}_{\mathrm{P}}
$$

where $k_{\text {on }}$ and $k_{\text {off }}$ are the reaction association and dissociation rates, respectively.

The governing eqs 3-5 are subjected to the no-flux boundary conditions at the ceiling of the device, on the top of the bulk solution $(z=0)$, and at the bottom of the pore $(z=$ $H+L_{\mathrm{p}}$ ) (see scheme in Figure 2), as well as the continuity of the concentration and the flux at the interface between the bulk and the pores $(z=H)$

$$
\begin{aligned}
\frac{\partial c_{\mathrm{A}, \mathrm{bulk}}}{\partial z}=0 & \text { at } z=0 \\
\frac{\partial c_{\mathrm{A}, \mathrm{PSi}}}{\partial z}=0 & \text { at } z=H+L_{\mathrm{p}}
\end{aligned}
$$



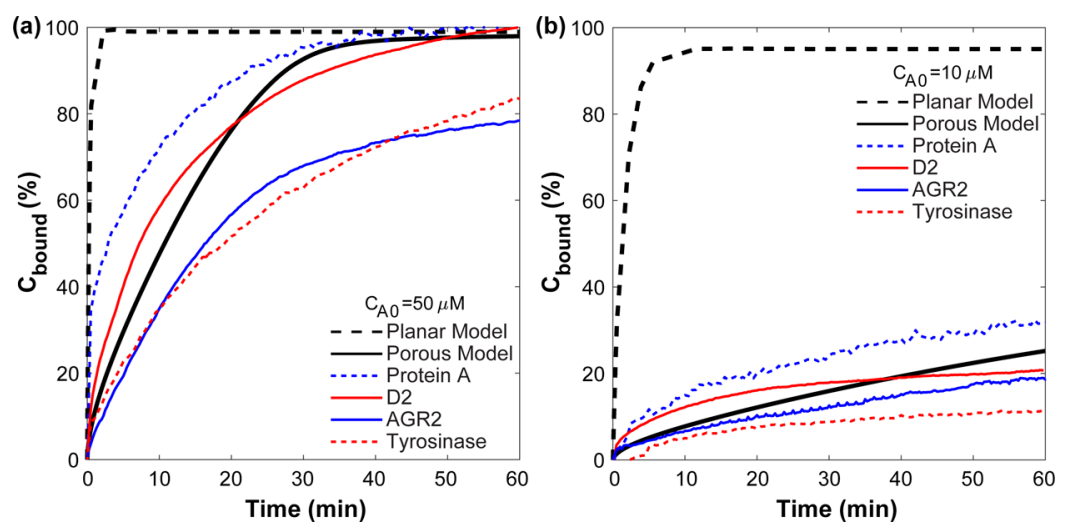

Figure 3. Comparison of experimental binding curves of the investigated aptasensors to numerical simulation results obtained for the porous and planar models, at target concentrations of (a) 50 and (b) $10 \mu \mathrm{M}$. For the experimental data, the EOT signals for each aptasensor were normalized to the maximal EOT signal obtained upon aptasensor saturation with the target. For simulated binding, the curves present the bound analyte, normalized to the probe concentration or density, at the bottom of the pore as a function of time.

$$
c_{\mathrm{A}, \text { bulk }}=c_{\mathrm{A}, \mathrm{PSi}} \quad \text { at } z=H
$$

and

$$
D_{\text {bulk }} \frac{\partial c_{\mathrm{A}, \text { bulk }}}{\partial z}=D_{\mathrm{PSi}} \frac{\partial c_{\mathrm{A}, \mathrm{PSi}}}{\partial z} \quad \text { at } z=H
$$

We assume that the initial analyte concentration in the bulk is $c_{\mathrm{A} 0}$ and that the initial concentrations of the analyte and of the immobilized analyte-probe complexes within the porous layer are both zero

$$
\begin{aligned}
& c_{\mathrm{A}, \mathrm{bulk}}(z, t=0)=c_{\mathrm{A} 0} \quad 0 \leq z \leq H \\
& c_{\mathrm{A}, \mathrm{PSi}}(z, t=0)=c_{\mathrm{A} 0}\left[(1-\theta(z)] \quad H \leq z \leq H+L_{\mathrm{p}}\right. \\
& c_{\mathrm{P}}(z, t=0)=0
\end{aligned}
$$

where the function $\theta(z)$ is defined as

$$
\theta(z)= \begin{cases}0 & z=H \\ 1 & z>H\end{cases}
$$

The governing eqs 3-5 are coupled through the boundary conditions (6)-(9) and should be solved together to obtain the concentration in the bulk and in the porous layer.

Within the porous layer, the constrained space of the pore leads to hindered diffusion of the analyte molecules. Thus, the diffusivity coefficient of the analyte within the PSi, $D_{\mathrm{PSi}}$, should be corrected according to the molecular and hindered diffusion phenomena, accounting for steric restriction, hindered Brownian motion, and energetic interactions of poresolvent-analyte. ${ }^{50}$ Empirical models for $D_{\mathrm{PSi}}$ describe the hindered diffusivity as a function of the parameter $\alpha$, defined as the ratio of the hydrodynamic diameter of the analyte $d_{\mathrm{A}}$ and the diameter of the average pore $d_{\mathrm{p}}(z, t)$, that is, $\alpha(z, t)=d_{\mathrm{A}} /$ $d_{\mathrm{p}}(z, t)$. The diameter of the average pore $d_{\mathrm{p}}(z, t)$ decreases upon binding of the analyte to the immobilized probes on the pore walls and can be related to the bound analyte concentration $c_{\mathrm{P}}$ through

$$
d_{\mathrm{p}}(z, t)=d_{\mathrm{p} 0}-2\left[\frac{c_{\mathrm{P}}}{c_{\mathrm{B} 0}} d_{\mathrm{A}}+d_{\mathrm{B}}\right]
$$

where $d_{\mathrm{p} 0}$ is the initial diameter of the pore and $d_{\mathrm{B}}$ is the hydrodynamic diameter of the probe. Thus, the ratio $\alpha$ is given by

$$
\alpha(z, t)=\frac{d_{\mathrm{A}}}{d_{\mathrm{p} 0}-2\left[\left(c_{\mathrm{P}} / c_{\mathrm{B} 0}\right) d_{\mathrm{A}}+d_{\mathrm{B}}\right]}
$$

For our study, we utilize the comprehensive model derived by Dechadilok and Deen ${ }^{54}$ for cylindrical pores for the estimation of $D_{\mathrm{PSi}}$

$$
\begin{aligned}
& D_{\mathrm{PSi}}(z, t)= \\
& D_{\text {bulk }}\left[\begin{array}{l}
1+\frac{9}{8} \alpha \ln \alpha-1.56034 \alpha+0.528155 \alpha^{2} \\
+1.91521 \alpha^{3} \\
-2.81903 \alpha^{4}+0.270788 \alpha^{5}+1.10115 \alpha^{6} \\
-0.435933 \alpha^{7}
\end{array}\right]
\end{aligned}
$$

Comparison of Theoretical Models to Experimental Results. We solve the three coupled nonlinear differential equations numerically, using parameters characteristic of actual PSi aptasensors, described in Table 1. Specifically, we use an average pore diameter value of $50 \mathrm{~nm}$, a porous layer thickness of $5.5 \mu \mathrm{m}$, and a bulk solution height of $1 \mathrm{~mm}$. A representative value of $\sim 7 \times 10^{-11} \mathrm{~m}^{2} \mathrm{~s}^{-1}$ is applied for the protein bulk diffusivity coefficient, based on the relative protein sizes. ${ }^{75}$ For the kinetic binding rate constants, we use those of the pair of anti-protein A aptamer and protein A, which were previously determined by SPR as $1.21 \times 10^{3}$ and $6.23 \times 10^{-4} \mathrm{~s}^{-1}$ for the association and dissociation rates, respectively. ${ }^{62}$ The aptamer and target protein diameters are 3 and $5.3 \mathrm{~nm}$, respectively. Please see Table S1 (Supporting Information) for a comprehensive summary of all values used for the numerical simulations.

We have experimentally determined the concentration of the immobilized aptamers within the porous layer, using a fluorescently labeled aptamer and its subsequent cleavage. This method was applied for the anti-AGR2 and anti-his tag aptasensors, and the resulting aptamer concentration ranges between $1.0 \pm 0.2$ and $6.28 \pm 0.06 \mathrm{mM}$, respectively (see Table S2 for detailed results, Supporting Information). These values provide an order of magnitude estimation for the probe concentration for all studied aptasensors, and thus, for the 

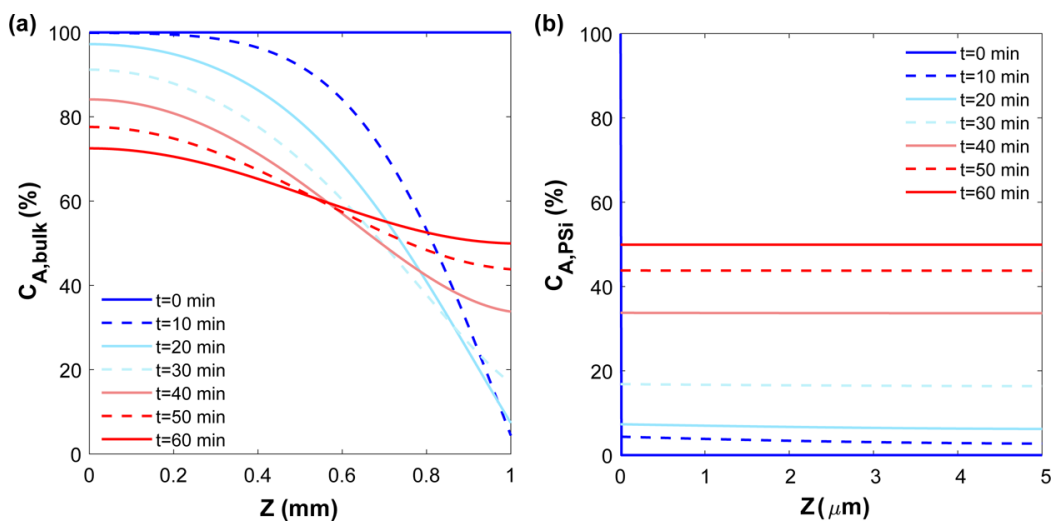

Figure 4. Simulation of the distribution of the target concentration in the $z$ axis at different time points in (a) the bulk solution and (b) the porous layer, obtained by the porous model. The target concentration is normalized to an initial target solution concentration of $50 \mu \mathrm{M}$.

numerical simulations, a representative value is used, that is, an aptamer concentration of $3.6 \mathrm{mM}$.

Figure $3 \mathrm{a}, \mathrm{b}$ depicts the real-time experimental binding curves of the investigated aptasensors for detection of the target proteins at concentrations of 50 and $10 \mu \mathrm{M}$, respectively, in comparison to results obtained by numerical simulations. We present the simulation results for the porous model and those obtained for a planar model, where the porous layer is assumed to be a perfect collector as is conventionally considered in the literature. ${ }^{28,58,59}$ For the planar model (see detailed derivation of the model in the Supporting Information), we applied the same reaction kinetic parameters and capture probe surface density, as for the porous model. For the experimental binding curves, the percentage of target binding was calculated by normalizing the EOT signal to the maximal EOT signal obtained at aptasensor saturation with the target. While our suggested porous model presents a relatively good fit to the experimental results at both studied target concentrations, the planar model highly overestimates the binding rate, even at a high target concentration where mass transfer limitations should be less pronounced (Figure 3a). Only at long enough times, the experimental and the model curves converge, while at (relatively) short times, the planar model greatly diverges. Moreover, these deviations intensify at low target concentrations, as shown in Figure $3 \mathrm{~b}$ for a target concentration of $10 \mu \mathrm{M}$ (and in Figure S1 for lower target concentrations of 1 and $0.5 \mu \mathrm{M}$, Supporting Information), mainly ascribed to the decrease in the concentration gradient, that is, diffusion driving force, for lower target concentrations. It should be emphasized that the porous model fits the experimental results also when applying other reaction rates, while the overestimation of the planar model is still apparent, as shown in Figure S2 (Supporting Information). Our results demonstrate that hindered diffusion has a major impact on the binding rates of PSi-based biosensors and cannot be neglected. Thus, the porous model is essential for accurate representation of the binding behavior, especially when studying relevant target concentrations, ${ }^{76}$ which are orders of magnitude lower than those presented here.

To illustrate the significance of each of the diffusion phenomena, we present, in Figure 4, the simulated distribution of the target concentration (in the $z$ axis) in the bulk solution and in the porous layer, at different time points. At the initial stage of binding, the target is rapidly depleted near the pore entry (to a value below $5 \%$ of the initial target concentration), and a diffusion boundary layer is formed, spanning deep into the bulk solution. With the progress of the diffusion of the target into the porous layer, the concentration gradient slowly diminishes, until equilibration is reached. In contrast, for a planar model, the depletion of the target on the biosensor surface is significantly lower, as shown in Figure S3 (Supporting Information). These results indicate that both diffusion processes, in the bulk and in the porous layer, are interrelated: the diffusion within the porous layer leads to a rapid and substantial formation of a diffusion boundary layer within the bulk solution. Thus, a similar binding behavior observed for the different studied biosensors (see Figure 1c) is ascribed to the mass transfer limitations and to the interconnected effect of both diffusion processes. These conceal the capture probe-target protein reaction, and our main conclusion from this study is that without significantly accelerating the mass transfer rate, the contribution of higher affinity capture probes for improving the biosensing performance (i.e., sensitivity and detection time) will be imperceptible. It should be kept in mind that the system noise also plays a critical role in determining the biosensor performance, and it should be minimized. Thus, when applying methods for mass transfer acceleration, the resulting LOD will also depend on their effect on the noise.

We further demonstrate the contribution of mass transfer acceleration to the enhancement of the apparent binding rate by application of mixing of the target solution on top of the biosensor. This results in a constant analyte concentration within the solution above the porous nanostructure, eliminating the diffusion gradient in the bulk solution and decreasing the diffusion path length to the porous layer. ${ }^{14}$ Figure S4 (Supporting Information) compares biosensing results with and without target mixing (10 min mixing followed by incubation $v$ s incubation only). During mixing, the EOT signal is observed to rapidly increase, and a significantly higher apparent binding rate (by $>5$ fold) is obtained in comparison to the nonmixed system, thus demonstrating the profound effect of mass transfer acceleration on enhancing the target flux into the porous layer. It should be noted that we use manual mixing in this work, while a better performance in terms of sample-to-sample reproducibility will be obtained upon mixing automation.

Effect of Biosensor Characteristics. We use the derived porous model to study the effect of important biosensor characteristics, which can be tailored during the biosensor construction, on the binding rate. The first parameter we examine is the capture probe surface density, which is 

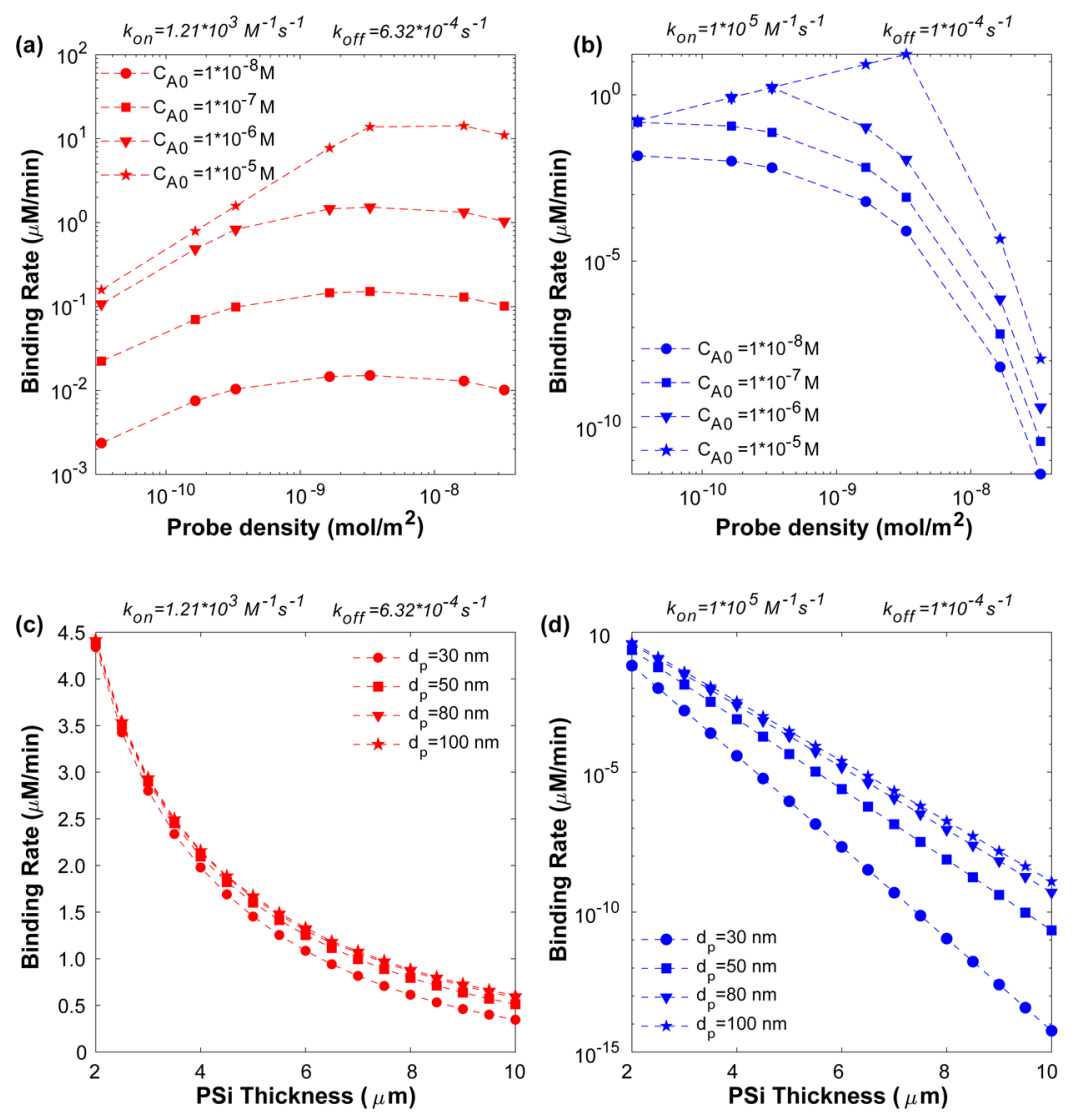

Figure 5. Effect of biosensor characteristics on the simulated target binding rate. (a,b) Effect of capture probe surface density for different target concentrations, for low $\left(k_{\text {on }}=1.21 \times 10^{3} \mathrm{M}^{-1} \mathrm{~s}^{-1}\right.$ and $\left.k_{\text {off }}=6.32 \times 10^{-4} \mathrm{~s}^{-1}\right)$ and high $\left(k_{\text {on }}=10^{5} \mathrm{M}^{-1} \mathrm{~s}^{-1}\right.$ and $\left.k_{\text {off }}=10^{-4} \mathrm{~s}^{-1}\right)$ affinity interactions, respectively. (c,d) Effect of porous silicon layer thickness for different pore diameters for a target concentration of $1 \mu \mathrm{M}$, for low $\left(k_{\text {on }}=1.21 \times 10^{3}\right.$ $\mathrm{M}^{-1} \mathrm{~s}^{-1}$ and $\left.k_{\text {off }}=6.32 \times 10^{-4} \mathrm{~s}^{-1}\right)$ and high $\left(k_{\mathrm{on}}=10^{5} \mathrm{M}^{-1} \mathrm{~s}^{-1}\right.$ and $\left.k_{\text {off }}=10^{-4} \mathrm{~s}^{-1}\right)$ affinity interactions, respectively. The binding rate was calculated as the slope of bound target concentration $v s$ time curve, in a time frame of $60 \mathrm{~min}$, at the bottom of the pore.

considered of high importance for surface-based biosensors, in general, $^{77-80}$ and PSi-based biosensors, in particular. ${ }^{22,23,81,82}$ Although for both planar and porous surfaces, an overall similar capture probe density may be attained, the large surface area of PSi allows immobilizing larger amounts of capture probes and their concentration within the nanoscale pores. This in turn reduces the binding time, affects the apparent off rate of the probe-analyte complexes, and enhances the biosensor sensitivity, as has been previously suggested. ${ }^{14,22,23}$ In the present work, we would like to investigate whether an excess of capture probes within the pores may lead to a counter effect on the mass transport rate. Figure $5 \mathrm{a}$ presents a simulation of the effect of capture probe surface density within the porous layer on the target binding rate for different analyte concentrations. We apply, in the simulations, a capture probe density range of $\sim 10^{-11}$ to $\sim 10^{-8} \mathrm{~mol} \mathrm{~m}{ }^{-2}$, which has been utilized in PSi-based biosensors. ${ }^{23,28,81}$ Increasing the capture probe surface density results in higher binding rates until an optimal surface density value; above this value, the binding rate slightly decreases. For a higher affinity interaction (Figure $5 b$ ), with an association rate of $10^{5} \mathrm{M}^{-1} \mathrm{~s}^{-1}$ and a dissociation rate of $10^{-4} \mathrm{~s}^{-1}$, which is characteristic for antibody-ligand interactions, ${ }^{43,83}$ increase in surface density results in a drastic decrease in the binding rate. We attribute this behavior to the decrease in the free porous volume available for the transport of the target. In addition, at a high probe density, a depletion region can rapidly build, which in turn will increase the thickness of the diffusion boundary layer in the bulk (adjacent to the pore entry), ${ }^{77}$ and as a result, both bulk and hindered diffusion rates will decrease. Our results suggest that mass transfer limitations require maintaining an optimum capture probe surface density, below a certain threshold, while considering immobilization levels that would produce a biosensor response with an acceptable signal-to-noise ratio. This has also high significance for maintaining active probes while immobilized, avoiding steric crowding effects. ${ }^{82,84}$

Two additional key parameters which can be easily tailored for PSi biosensors are the porous layer thickness and pore diameter. These affect both the optical properties of the nanostructure and the hindered diffusion within the pores, as they dictate the available free porous volume for molecular transport. ${ }^{20,40,41,51}$ Figure $5 \mathrm{c}$ presents the effect of porous layer thickness and pore diameter on the binding rate (as simulated at the bottom of the porous layer), while the capture probe and the analyte concentrations are kept constant. The results show a significant effect of the porous layer thickness on the binding rate, with the latter decreasing for increasing thickness. This agrees with other studies and is related to the increase in diffusion time inside the porous layer with increasing pore length, which scales as $t_{\mathrm{d}} \propto L_{\mathrm{p}}^{2} / D_{\mathrm{PSi}}{ }^{35,36}$ Thus, decreasing the PSi layer thickness will result in an improved biosensor sensitivity. ${ }^{35}$ The impact of the pore diameter (in the range of $30-100 \mathrm{~nm}$ ) on the binding rates is less pronounced. However, this result is valid for aptasensors, where the size of the capture probe is significantly lower than the diameter of 
the pores (see Table S2), whereas for larger probes (e.g., antibodies), this pore diameter range will be narrow. ${ }^{33,82}$

When higher affinity interaction parameters are used for the simulation (an association rate of $10^{5} \mathrm{M}^{-1} \mathrm{~s}^{-1}$ and a dissociation rate of $10^{-4} \mathrm{~s}^{-1}$, typical for antibody-ligand interactions), the effect of the layer thickness and pore diameter is intensified, see Figure 5d. The simulated binding rate decreases by orders of magnitude for the thicker porous layer or smaller pore diameter. This is related to the impact of the mass transfer limitation in the bulk solution and the rapid formation of a depletion region at the pore entrance, owing to the fast uptake of the target. Thus, for biosensing interactions with higher affinity, the porous layer should be designed with smaller thickness and larger pore diameter, compared to lower affinity interactions. The number of pores in the PSi nanostructure, which can be correlated to the PSi porosity (see derivation in the Supporting Information), also characterizes the porous layer and influences the target capture rate. As the number of pores, and accordingly the porosity, decreases, a higher binding rate is observed, as presented in Figure S5 (Supporting Information). This is related to the higher diffusion flux into each pore. Nevertheless, the effect is less pronounced compared to the porous layer thickness and the pore diameter, even for a high affinity interaction (see Figure S5b, Supporting Information).

It should be kept in mind that the reflectivity of the PSi transducer, in terms of the intensity and number of fringes (for RIFTS), highly depends on the pore diameter and the thickness of the porous layer. ${ }^{20,40,41}$ Figure S6 (Supporting Information) shows the experimental reflectivity spectra for PSi films of different thicknesses, showing the decrease in the number of fringes for thinner porous films. This in turn affects RIFTS signal processing, whereas the reflectivity of a layer with a thickness below $1 \mu \mathrm{m}$ cannot be reliably analyzed. Thus, the porous layer thickness should be as low as possible to allow high binding rates and reflectance intensity, but this value should be optimized to yield a sufficient number of fringes. The latter is also dependent on the pore diameter. ${ }^{39-41}$ The effect of the PSi structural characteristics on the optical properties is interrelated; thus, their collective contribution should be considered upon nanostructure optimization. ${ }^{18,40}$ To highlight the importance of a rational biosensor design, Figure S7 presents the simulated binding rate for various target concentrations, upon decreasing the porous layer thickness to $2 \mu \mathrm{m}$ and the capture probe density to $2.3 \times 10^{-9} \mathrm{~mol} \mathrm{~m}^{-2}$, compared to the original PSi aptasensor. These values have been chosen according to the simulation results of the effect of the PSi thickness and capture probe density on the target binding rate, as presented in Figure 5. A significantly enhanced binding rate is observed, suggesting that the LOD can be improved by at least 10 -fold by simply adjusting the biosensor characteristics.

\section{CONCLUSIONS}

A theoretical model, in which the complex mass transfer processes involved in target capture within PSi-based transducers, is derived. The model considers the bulk diffusion of the target in the solution toward the biosensor surface, the hindered diffusion within the porous layer, and simultaneous reaction with the immobilized capture probe molecules. We solve the model numerically using parameters which were derived experimentally and are characteristic of PSi-based biosensors. The model successfully captures the target binding rates of several PSi aptasensors designed for protein detection, while the common-practiced model, in which the PSi is assumed as a planar surface and thus neglects the hindered diffusion phenomenon, drastically overestimates the target binding rate. Numerical simulation results indicate an interrelated effect of both diffusion processes, in the bulk solution and in the porous layer, which cannot be separated. Thus, diffusion within the porous layer should not be neglected, and both diffusion phenomena are important to accurately represent the transport within PSi-based biosensors, especially at low target concentrations. The model results can explain ours and others encountered low sensitivity of PSi biosensors (in the micromolar range), and similar target capture regardless of the nature of the capture probe-target pair and their theoretical binding affinity. Thus, accelerating mass transport, while maintaining similar (or lower) noise levels, is essential in order to exploit the advantages of high affinity capture probes. It should be emphasized that although we focus, in our work, on mass transfer limitations, system noise and signal processing methods also affect the performance of the biosensor and should be considered for obtaining maximal enhancement of the biosensor.

The proposed theoretical model is used to investigate the effect of PSi biosensor characteristics, that is, capture probe surface density, porous layer thickness, and pore diameter, which can be tailored during biosensor construction, on the target capture rate. Importantly, we show that the increased surface area of the PSi, which is one of the main advantages of these nanostructured transducers, can in turn lead to an excess of target binding sites. This results in further diffusion impedance in the bulk solution and the porous layer. Thus, the amount of immobilized capture probes and the corresponding surface density should be optimized to allow an efficient mass transfer rate, while still producing a biosensor response with a reliable signal-to-noise ratio. Porous layer thickness and pore diameter have also high impact on the binding rate, the latter decreasing for thicker PSi layers and smaller pore diameters. However, the pore diameter should be large enough to accommodate the bioreceptor and the target molecules, while the porous layer thickness should be thinned, while allowing a reliable optical signal processing.

\section{ASSOCIATED CONTENT}

\section{Supporting Information}

The Supporting Information is available free of charge at https://pubs.acs.org/doi/10.1021/acssensors.0c00670.

Derivation of a planar model, fitting parameters used for numerical simulations, results of quantification of immobilized aptamers, comparison of experimental results to model simulations for different binding affinity interactions, change in target concentration in the $z$ axis simulated by the planar model, and characterization of PSi with different layer thicknesses (PDF)

\section{AUTHOR INFORMATION}

\section{Corresponding Author}

Ester Segal - Department of Biotechnology and Food Engineering and The Russell Berrie Nanotechnology Institute, Technion-Israel Institute of Technology, Haifa 3200003, Israel; @ orcid.org/0000-0001-9472-754X; Email: esegal@ technion.ac.il 


\section{Authors}

Sofia Arshavsky Graham - Department of Biotechnology and Food Engineering, Technion-Israel Institute of Technology, Haifa 3200003, Israel; Institute of Technical Chemistry, Leibniz Universität Hannover, Hanover 30167, Germany

Evgeniy Boyko - Department of Mechanical Engineering, Technion-Israel Institute of Technology, Haifa 3200003, Israel

Rachel Salama - Department of Biotechnology and Food Engineering, Technion-Israel Institute of Technology, Haifa 3200003, Israel

Complete contact information is available at: https://pubs.acs.org/10.1021/acssensors.0c00670

\section{Author Contributions}

All authors have given approval to the final version of the manuscript.

\section{Notes}

The authors declare no competing financial interest.

\section{ACKNOWLEDGMENTS}

This work was supported by the ISRAEL SCIENCE FOUNDATION (grant no. 704/17) and the German Research Foundation under the grant SCHE 279/32-1. We thank Prof. Moran Bercovici for his valuable support in the theoretical studies. We thank Prof. Ayelet Fishman and her group and Prof. Yuval Shoham and his group at the Department for Biotechnology and Food Engineering at the Technion-Israel Institute of Technology for supplying target proteins. S.A.G. is most grateful for the Russell Berrie Nanotechnology Excellence Scholarship for Graduate Students. E.B. is supported by the Adams Fellowship Program of the Israel Academy of Sciences and Humanities.

\section{REFERENCES}

(1) Janshoff, A.; Galla, H.-J.; Steinem, C. Piezoelectric Mass-Sensing Devices as Biosensors-An Alternative to Optical Biosensors? Angew. Chem. 2000, 39, 4004-4032.

(2) McPhillips, J.; Murphy, A.; Jonsson, M. P.; Hendren, W. R.; Atkinson, R.; Höök, F.; Zayats, A. V.; Pollard, R. J. High-Performance Biosensing Using Arrays of Plasmonic Nanotubes. ACS Nano 2010, 4, 2210-2216.

(3) Guo, X. Surface plasmon resonance based biosensor technique: A review. J. Biophot. 2012, 5, 483-501.

(4) Länge, K.; Rapp, B. E.; Rapp, M. Surface acoustic wave biosensors: a review. Anal. Bioanal. Chem. 2008, 391, 1509-1519.

(5) Fan, X.; White, I. M.; Shopova, S. I.; Zhu, H.; Suter, J. D.; Sun, Y. Sensitive optical biosensors for unlabeled targets: A review. Anal. Chim. Acta 2008, 620, 8-26.

(6) Squires, T. M.; Messinger, R. J.; Manalis, S. R. Making it stick: convection, reaction and diffusion in surface-based biosensors. Nat. Biotechnol. 2008, 26, 417-426.

(7) Schuck, P.; Minton, A. P. Analysis of Mass Transport-Limited Binding Kinetics in Evanescent Wave Biosensors. Anal. Biochem. 1996, 240, 262-272.

(8) Nair, P. R.; Alam, M. A. Performance limits of nanobiosensors. Appl. Phys. Lett. 2006, 88, 233120.

(9) Sheehan, P. E.; Whitman, L. J. Detection Limits for Nanoscale Biosensors. Nano Lett. 2005, 5, 803-807.

(10) Lynn, N. S.; Śípová, H.; Adam, P.; Homola, J. Enhancement of affinity-based biosensors: effect of sensing chamber geometry on sensitivity. Lab Chip 2013, 13, 1413-1421.

(11) Schuck, P. Kinetics of ligand binding to receptor immobilized in a polymer matrix, as detected with an evanescent wave biosensor. I.
A computer simulation of the influence of mass transport. Biophys. J. 1996, 70, 1230-1249.

(12) O'Shannessy, D. J.; Winzor, D. J. Interpretation of Deviations from Pseudo-First-Order Kinetic Behavior in the Characterization of Ligand Binding by Biosensor Technology. Anal. Biochem. 1996, 236, $275-283$.

(13) Hu, G.; Gao, Y.; Li, D. Modeling micropatterned antigenantibody binding kinetics in a microfluidic chip. Biosens. Bioelectron. 2007, 22, 1403-1409.

(14) Wu, Y.; Tilley, R. D.; Gooding, J. J. Challenges and Solutions in Developing Ultrasensitive Biosensors. J. Am. Chem. Soc. 2019, 141, $1162-1170$.

(15) Arshavsky-Graham, S.; Massad-Ivanir, N.; Segal, E.; Weiss, S. Porous Silicon-Based Photonic Biosensors: Current Status and Emerging Applications. Anal. Chem. 2019, 91, 441-467.

(16) Tieu, T.; Alba, M.; Elnathan, R.; Cifuentes-Rius, A.; Voelcker, N. H. Advances in Porous Silicon-Based Nanomaterials for Diagnostic and Therapeutic Applications. Adv. Ther. 2019, 2, 1800095.

(17) Lin, V. S.; Motesharei, K.; Dancil, K.-P. S.; Sailor, M. J.; Ghadiri, M. R. A Porous Silicon-Based Optical Interferometric Biosensor. Science 1997, 278, 840-843.

(18) Sailor, M. J. Porous Silicon in Practice: Preparation, Characterization and Applications; John Wiley \& Sons, 2012.

(19) Pacholski, C.; Yu, C.; Miskelly, G. M.; Godin, D.; Sailor, M. J. Reflective Interferometric Fourier Transform Spectroscopy: A SelfCompensating Label-Free Immunosensor Using Double-Layers of Porous SiO2. J. Am. Chem. Soc. 2006, 128, 4250-4252.

(20) Pacholski, C.; Sartor, M.; Sailor, M. J.; Cunin, F.; Miskelly, G. M. Biosensing Using Porous Silicon Double-Layer Interferometers: Reflective Interferometric Fourier Transform Spectroscopy. J. Am. Chem. Soc. 2005, 127, 11636-11645.

(21) Rodriguez, G. A.; Hu, S.; Weiss, S. M. Porous silicon ring resonator for compact, high sensitivity biosensing applications. Opt. Express 2015, 23, 7111-7119.

(22) DeLouise, L. A.; Kou, P. M.; Miller, B. L. Cross-Correlation of Optical Microcavity Biosensor Response with Immobilized Enzyme Activity. Insights into Biosensor Sensitivity. Anal. Chem. 2005, 77, $3222-3230$

(23) Hu, S.; Zhao, Y.; Qin, K.; Retterer, S. T.; Kravchenko, I. I.; Weiss, S. M. Enhancing the Sensitivity of Label-Free Silicon Photonic Biosensors through Increased Probe Molecule Density. ACS Photonics 2014, 1, 590-597.

(24) Gupta, B.; Zhu, Y.; Guan, B.; Reece, P. J.; Gooding, J. J. Functionalised porous silicon as a biosensor: emphasis on monitoring cells in vivo and in vitro. Analyst 2013, 138, 3593-3615.

(25) Mariani, S.; Strambini, L. M.; Barillaro, G. Electrical Double Layer-Induced Ion Surface Accumulation for Ultrasensitive Refractive Index Sensing with Nanostructured Porous Silicon Interferometers. ACS Sens. 2018, 3, 595-605.

(26) Mariani, S.; Pino, L.; Strambini, L. M.; Tedeschi, L.; Barillaro, G. 10000 -Fold Improvement in Protein Detection Using Nanostructured Porous Silicon Interferometric Aptasensors. ACS Sens. 2016, 1, 1471-1479.

(27) Mariani, S.; Strambini, L. M.; Barillaro, G. Femtomole Detection of Proteins Using a Label-Free Nanostructured Porous Silicon Interferometer for Perspective Ultrasensitive Biosensing. Anal. Chem. 2016, 88, 8502-8509.

(28) Zhao, Y.; Gaur, G.; Retterer, S. T.; Laibinis, P. E.; Weiss, S. M. Flow-Through Porous Silicon Membranes for Real-Time Label-Free Biosensing. Anal. Chem. 2016, 88, 10940-10948.

(29) Mariani, S.; Robbiano, V.; Strambini, L. M.; Debrassi, A.; Egri, G.; Dähne, L.; Barillaro, G. Layer-by-layer biofunctionalization of nanostructured porous silicon for high-sensitivity and high-selectivity label-free affinity biosensing. Nat. Commun. 2018, 9, 5256.

(30) Vilensky, R.; Bercovici, M.; Segal, E. Oxidized Porous Silicon Nanostructures Enabling Electrokinetic Transport for Enhanced DNA Detection. Adv. Funct. Mater. 2015, 25, 6725-6732.

(31) Arshavsky-Graham, S.; Massad-Ivanir, N.; Paratore, F.; Scheper, T.; Bercovici, M.; Segal, E. On Chip Protein Pre-Concentration for 
Enhancing the Sensitivity of Porous Silicon Biosensors. ACS Sens. 2017, 2, 1767-1773.

(32) Rong, G.; Najmaie, A.; Sipe, J. E.; Weiss, S. M. Nanoscale porous silicon waveguide for label-free DNA sensing. Biosens. Bioelectron. 2008, 23, 1572-1576.

(33) Rong, G.; Weiss, S. M. Biomolecule size-dependent sensitivity of porous silicon sensors. Phys. Status Solidi A 2009, 206, 1365-1368. (34) Anderson, M. A.; Tinsley-Bown, A.; Allcock, P.; Perkins, E. A.; Snow, P.; Hollings, M.; Smith, R. G.; Reeves, C.; Squirrell, D. J.; Nicklin, S.; Cox, T. I. Sensitivity of the optical properties of porous silicon layers to the refractive index of liquid in the pores. Phys. Status Solidi A 2003, 197, 528-533.

(35) Zhu, W.; Wang, C.; Wang, H.; Li, G. Theory and simulation of diffusion-adsorption into a molecularly imprinted mesoporous film and its nanostructured counterparts. Experimental application for trace explosive detection. RSC Adv. 2014, 4, 40676-40685.

(36) Kant, K.; Yu, J.; Priest, C.; Shapter, J. G.; Losic, D. Impedance nanopore biosensor: influence of pore dimensions on biosensing performance. Analyst 2014, 139, 1134-1140.

(37) Lu, S.; Song, Z.; He, J. Diffusion-Controlled Protein Adsorption in Mesoporous Silica. J. Phys. Chem. B 2011, 115, 7744-7750.

(38) DeLouise, L. A.; Miller, B. L. Quantatitive Assessment of Enzyme Immobilization Capacity in Porous Silicon. Anal. Chem. 2004, 76, 6915-6920.

(39) Macias, G.; Ferré-Borrull, J.; Pallarès, J.; Marsal, L. F. Effect of pore diameter in nanoporous anodic alumina optical biosensors. Analyst 2015, 140, 4848-4854.

(40) Kumeria, T.; Losic, D. Controlling interferometric properties of nanoporous anodic aluminium oxide. Nanoscale Res. Lett. 2012, 7, 88.

(41) Tinsley-Bown, A. M.; Canham, L. T.; Hollings, M.; Anderson, M. H.; Reeves, C. L.; Cox, T. I.; Nicklin, S.; Squirrell, D. J.; Perkins, E.; Hutchinson, A.; Sailor, M. J.; Wun, A. Tuning the Pore Size and Surface Chemistry of Porous Silicon for Immunoassays. Phys. Status Solidi A 2000, 182, 547-553.

(42) Zhao, Y.; Gaur, G.; Mernaugh, R. L.; Laibinis, P. E.; Weiss, S. M. Comparative Kinetic Analysis of Closed-Ended and Open-Ended Porous Sensors. Nanoscale Res. Lett. 2016, 11, 395.

(43) Schwartz, M. P.; Alvarez, S. D.; Sailor, M. J. Porous SiO2Interferometric Biosensor for Quantitative Determination of Protein Interactions: Binding of Protein A to Immunoglobulins Derived from Different Species. Anal. Chem. 2007, 79, 327-334.

(44) Martin, F.; Walczak, R.; Boiarski, A.; Cohen, M.; West, T.; Cosentino, C.; Ferrari, M. Tailoring width of microfabricated nanochannels to solute size can be used to control diffusion kinetics. J. Controlled Release 2005, 102, 123-133.

(45) Bouchaud, J.-P.; Georges, A. Anomalous diffusion in disordered media: Statistical mechanisms, models and physical applications. Phys. Rep. 1990, 195, 127-293.

(46) Renkin, E. M. FILTRATION, DIFFUSION, AND MOLECULAR SIEVING THROUGH POROUS CELLULOSE MEMBRANES. J. Gen. Physiol. 1954, 38, 225-243.

(47) Krishna, R. Describing the Diffusion of Guest Molecules Inside Porous Structures. J. Phys. Chem. C 2009, 113, 19756-19781.

(48) Satterfield, C. N.; Colton, C. K.; Pitcher, W. H., Jr Restricted diffusion in liquids within fine pores. AIChE J. 1973, 19, 628-635.

(49) Saltzman, W. M.; Langer, R. Transport rates of proteins in porous materials with known microgeometry. Biophys. J. 1989, 55, $163-171$.

(50) Deen, W. M. Hindered transport of large molecules in liquidfilled pores. AIChE J. 1987, 33, 1409-1425.

(51) Mares, J. W.; Weiss, S. M. Diffusion dynamics of small molecules from mesoporous silicon films by real-time optical interferometry. Appl. Opt. 2011, 50, 5329-5337.

(52) Zürner, A.; Kirstein, J.; Döblinger, M.; Bräuchle, C.; Bein, T. Visualizing single-molecule diffusion in mesoporous materials. Nature 2007, 450, 705-708.

(53) Rühle, B.; Davies, M.; Lebold, T.; Bräuchle, C.; Bein, T. Highly Oriented Mesoporous Silica Channels Synthesized in Microgrooves and Visualized with Single-Molecule Diffusion. ACS Nano 2012, 6, $1948-1960$

(54) Dechadilok, P.; Deen, W. M. Hindrance Factors for Diffusion and Convection in Pores. Ind. Eng. Chem. Res. 2006, 45, 6953-6959.

(55) De Stefano, L.; Orabona, E.; Lamberti, A.; Rea, I.; Rendina, I. Microfluidics assisted biosensors for label-free optical monitoring of molecular interactions. Sens. Actuators, B 2013, 179, 157-162.

(56) Rea, I.; Orabona, E.; Lamberti, A.; Rendina, I.; De Stefano, L. A microfluidics assisted porous silicon array for optical label-free biochemical sensing. Biomicrofluidics 2011, 5, 034120.

(57) Marbán, G.; Ramírez-Montoya, L. A.; García, H.; Menéndez, J. Á.; Arenillas, A.; Montes-Morán, M. A. Load-dependent surface diffusion model for analyzing the kinetics of protein adsorption onto mesoporous materials. J. Colloid Interface Sci. 2018, 511, 27-38.

(58) Lazzara, T. D.; Mey, I.; Steinem, C.; Janshoff, A. Benefits and Limitations of Porous Substrates as Biosensors for Protein Adsorption. Anal. Chem. 2011, 83, 5624-5630.

(59) Wei, X.; Mares, J. W.; Gao, Y.; Li, D.; Weiss, S. M. Biomolecule kinetics measurements in flow cell integrated porous silicon waveguides. Biomed. Opt. Express 2012, 3, 1993-2003.

(60) Wu, J.; Wang, C.; Li, X.; Song, Y.; Wang, W.; Li, C.; Hu, J.; Zhu, Z.; Li, J.; Zhang, W.; Lu, Z.; Yang, C. J. Identification, Characterization and Application of a G-Quadruplex Structured DNA Aptamer against Cancer Biomarker Protein Anterior Gradient Homolog 2. PLoS One 2012, 7, No. e46393.

(61) Doyle, S. A.; Murphy, M. B. Aptamers and Methods for Their In Vitro Selection and Uses Thereof. U.S. Patent 0,142,582 A1, 2005.

(62) Stoltenburg, R.; Schubert, T.; Strehlitz, B. In vitro Selection and Interaction Studies of a DNA Aptamer Targeting Protein A. PLoS One 2015, 10, No. e0134403.

(63) Kökpinar, Ö.; Walter, J.-G.; Shoham, Y.; Stahl, F.; Scheper, T. Aptamer-based downstream processing of his-tagged proteins utilizing magnetic beads. Biotechnol. Bioeng. 2011, 108, 2371-2379.

(64) Nieba, L.; Krebber, A.; Plückthun, A. Competition BIAcore for Measuring True Affinities: Large Differences from Values Determined from Binding Kinetics. Anal. Biochem. 1996, 234, 155-165.

(65) Kilian, K. A.; Böcking, T.; Gooding, J. J. The importance of surface chemistry in mesoporous materials: lessons from porous silicon biosensors. Chem. Commun. 2009, 630-640.

(66) Urmann, K.; Walter, J.-G.; Scheper, T.; Segal, E. Label-Free Optical Biosensors Based on Aptamer-Functionalized Porous Silicon Scaffolds. Anal. Chem. 2015, 87, 1999-2006.

(67) Urmann, K.; Reich, P.; Walter, J.-G.; Beckmann, D.; Segal, E.; Scheper, T. Rapid and label-free detection of protein a by aptamertethered porous silicon nanostructures. J. Biotechnol. 2017, 257, 171177.

(68) Gaur, G.; Koktysh, D. S.; Weiss, S. M. Immobilization of Quantum Dots in Nanostructured Porous Silicon Films: Characterizations and Signal Amplification for Dual-Mode Optical Biosensing. Adv. Funct. Mater. 2013, 23, 3604-3614.

(69) Massad-Ivanir, N.; Shtenberg, G.; Tzur, A.; Krepker, M. A.; Segal, E. Engineering Nanostructured Porous $\mathrm{SiO} 2$ Surfaces for Bacteria Detection via "Direct Cell Capture". Anal. Chem. 2011, 83, 3282-3289.

(70) Kurganov, B. I.; Lobanov, A. V.; Borisov, I. A.; Reshetilov, A. N. Criterion for Hill equation validity for description of biosensor calibration curves. Anal. Chim. Acta 2001, 427, 11-19.

(71) Hill, A. V. PROCEEDINGS OF THE PHYSIOLOGICAL SOCIETY: January 22, 1910. J. Physiol. 1910, 40, i-vii.

(72) Tenenbaum, E.; Ben-Dov, N.; Segal, E. Tethered Lipid Bilayers within Porous Si Nanostructures: A Platform for (Optical) Real-Time Monitoring of Membrane-Associated Processes. Langmuir 2015, 31, 5244-5251.

(73) Massad-Ivanir, N.; Shtenberg, G.; Raz, N.; Gazenbeek, C.; Budding, D.; Bos, M. P.; Segal, E. Porous Silicon-Based Biosensors: Towards Real-Time Optical Detection of Target Bacteria in the Food Industry. Sci. Rep. 2016, 6, 38099. 
(74) Wu, C.; Rong, G.; Xu, J.; Pan, S.; Zhu, Y. Physical analysis of the response properties of porous silicon microcavity biosensor. Phys. E 2012, 44, 1787-1791.

(75) Tyn, M. T.; Gusek, T. W. Prediction of diffusion coefficients of proteins. Biotechnol. Bioeng. 1990, 35, 327-338.

(76) Kelley, S. O. What Are Clinically Relevant Levels of Cellular and Biomolecular Analytes? ACS Sens. 2017, 2, 193-197.

(77) Sikavitsas, V.; Nitsche, J. M.; Mountziaris, T. J. Transport and Kinetic Processes Underlying Biomolecular Interactions in the BIACORE Optical Biosensor. Biotechnol. Prog. 2002, 18, 885-897.

(78) Morton, T. A.; Myszka, D. G. Kinetic analysis of macromolecular interactions using surface plasmon resonance biosensors. In Methods in Enzymology; Academic Press, 1998; Vol. 295, pp 268-294.

(79) Myszka, D. G. Kinetic analysis of macromolecular interactions using surface plasmon resonance biosensors. Curr. Opin. Biotechnol. 1997, 8, 50-57.

(80) Peterson, A. W.; Heaton, R. J.; Georgiadis, R. M. The effect of surface probe density on DNA hybridization. Nucleic Acids Res. 2001, $29,5163-5168$

(81) Terracciano, M.; De Stefano, L.; Borbone, N.; Politi, J.; Oliviero, G.; Nici, F.; Casalino, M.; Piccialli, G.; Dardano, P.; Varra, M.; Rea, I. Solid phase synthesis of a thrombin binding aptamer on macroporous silica for label free optical quantification of thrombin. RSC Adv. 2016, 6, 86762-86769.

(82) Bonanno, L. M.; DeLouise, L. A. Steric Crowding Effects on Target Detection in an Affinity Biosensor. Langmuir 2007, 23, 58175823.

(83) Karlsson, R.; Michaelsson, A.; Mattsson, L. Kinetic analysis of monoclonal antibody-antigen interactions with a new biosensor based analytical system. J. Immunol. Methods 1991, 145, 229-240.

(84) Urmann, K.; Modrejewski, J.; Scheper, T.; Walter Johanna, G. Aptamer-modified nanomaterials: principles and applications. BioNanoMaterials 2017, 18, 20160012. 